\title{
Tanshinone IIA Reverses Gefitinib-Resistance In Human Non-Small-Cell Lung Cancer Via Regulation Of VEGFR/Akt Pathway
}

This article was published in the following Dove Press journal: OncoTargets and Therapy

\section{Rui Wang \\ Zhilin Luo \\ Hong Zhang \\ Tianhu Wang}

Department of Respiratory Disease Center, The Third Affiliated Hospital of Chongqing Medical University, Chongqing 40I I 20, People's Republic of China
Correspondence: Tianhu Wang Department of Respiratory Disease Center, The Third Affiliated Hospital of Chongqing Medical University, No.I Shuanghu Road, Yubei District, Chongqing 40I I20, People's Republic of China Email tianhuwang_cmu@I63.com
Background: Gefitinib-resistance is a primary obstacle for the treatment of non-small-cell lung cancer (NSCLC). It has been shown that tanshinone IIA (Tan IIA) could induce apoptosis of NSCLC cells. However, the role of combination of gefitinib with Tan IIA on gefitinib-resistance NSCLC cells remains unclear. Thus, this study aimed to investigate the role of combination on the proliferation, apoptosis and invasion of gefitinib-resistance NSCLC cells.

Methods: CCK-8, flow cytometric and transwell assays were applied to detect proliferation, apoptosis and invasion in gefitinib-resistance NSCLC cells, respectively. In addition, Western blotting assay was used to detect the expressions of p-EGFR, p-VEGFR2, and p-Akt in HCC827/gefitinib cells.

Results: In this study, Tan IIA enhanced the cytotoxic effect of gefitinib in gefitinibresistance NSCLC cells. In addition, the inhibitory effects of gefitinib on the proliferation, migration and invasion of gefitinib-resistance NSCLC cells were enhanced in the presence of Tan IIA. Moreover, Tan IIA enhanced the pro-apoptotic effect of gefitinib in gefitinibresistance NSCLC cells via increasing the level of cleaved caspase 3. Meanwhile, Tan IIA enhanced the sensitivity of HCC827/gefitinib cells to gefitinib via downregulation of the VEGFR2/Akt pathway. In vivo experiments further confirmed that combination of gefitinib with Tan IIA inhibited tumor growth in mouse xenograft model of HCC827/gefitinib.

Conclusion: We found that Tan IIA could enhance gefitinib sensitivity in gefitinib-resistance NSCLC cells. Therefore, combination of gefitinib with Tan IIA might be considered as a therapeutic approach for the treatment of gefitinib-resistant NSCLC.

Keywords: non-small-cell lung cancer, tanshinone IIA, gefitinib-resistance, VEGFR

\section{Introduction}

Lung cancer is the most common malignancy and the primary cause of cancerrelated death. ${ }^{1}$ Non-small cell lung carcinoma (NSCLC) comprises nearly $85 \%$ of all lung cancers. ${ }^{2,3}$ About $65 \%$ of patients with NSCLC were aggravated to advanced stage at the first diagnosis, which has been reported to show a poor prognosis with 1-year survival rate of only $30 \%$ to $40 \%$ after surgery. ${ }^{4}$ Recent years, surgical resection, radiotherapy and chemotherapy have been achieved promising benefit for patients with NSCLC. ${ }^{1,5,6}$ However, the prognosis of patients with NSCLC remains unsatisfactory due to the aggressiveness and metastasis of NSCLC. ${ }^{7}$ Thus, explore new treatment options for NSCLC are extremely necessary.

Gefitinib, an orally, epidermal growth factor receptor (EGFR) tyrosine kinase inhibitor, which could effectively inhibit the metastasis of NSCLC. ${ }^{8}$ Recently, it has 
been shown that some anti-tumor drugs, such as gefitinib and bevacizumab, exhibited anti-tumor effect via targeting inhibition of either the EGFR or vascular endothelial growth factor receptor (VEGFR) signaling pathways. ${ }^{9}$ EGFR and VEGFR signaling play important roles in tumor growth, angiogenesis and metastasis. ${ }^{10}$ Inhibiting both EGFR and VEGFR signaling might be a potential strategy for the treatment of NSCLC. Previous studies have established the relationship between EGFR and VEGFR2 signaling: inhibition of VEGFR2 signaling could enhance the anti-tumor effect of EGFR inhibitors. ${ }^{11,12}$ Gefitinib are high-targeting and lowtoxicity in advanced patients with NSCLC. ${ }^{13}$ It has been shown that gefitinib plays an important role in delaying NSCLC recurrence. ${ }^{14}$ Unfortunately, drug resistance was also accompanied, which severely weakened the clinical effectiveness of gefitinib. ${ }^{15}$ Recently, the bioactive components in traditional Chinese medicine (TCM) have been found to exhibit synergistically anti-tumor effect against NSCLC when combined with gefitinib. ${ }^{16}$ Thus, combination of TCM with gefitinib might be a potential therapeutic option for the treatment of NSCLC.

Tanshinone IIA (Tan IIA) is a lipophilic components deriving from the roots of Chinese herbs medicine Salvia miltiorrhiza Bunge. ${ }^{17}$ Tan IIA has been found to possess a number of biological activities, such as anti-inflammation, anti-oxidant and anti-tumor activities. ${ }^{18-20}$ Liu et al found that Tan IIA significantly inhibited the tumor growth of A549 human NSCLC xenografts. ${ }^{21}$ Meanwhile, Tan IIA could induce apoptosis in NSCLC cells via downregulation the level of p-Akt. ${ }^{22}$ However, the role of Tan IIA in gefitinib-resistanceof NSCLC remains unknown. Therefore, this study aimed to investigate the anti-tumor effects of combination of gefitinib with Tan IIA in gefitinib-resistant human NSCLC cells.

\section{Materials And Methods Cell Culture}

The NSCLC cell lines HCC827 and PC-9 were purchased from American Type Culture Collection (ATCC, Rockville, MD, USA). Two gefitinib-resistant cell lines (HCC827/ gefitinib and PC-9/gefitinib) were established by continuous exposure of NSCLC cells to a stepwise gradually concentration of gefitinib for more than 7 months. The NSCLC cells were cultured in Dulbecco's modified Eagle's medium (DMEM, Thermo Fisher Scientific, Waltham, MA, USA) supplemented with $10 \%$ heat-inactivated fetal bovine serum (FBS, Thermo Fisher Scientific) and 1\% penicillin-streptomycin at $37^{\circ} \mathrm{C}$ in a humidified atmosphere of $5 \% \mathrm{CO}_{2}$.
Gefitinib (cat. no. SML 1657) and Tan IIA (cat. no. T4952) were obtained from Sigma Aldrich (St. Louis, MO, USA).

\section{CCK-8 Assay}

The cell viability was detected by using the Cell Counting Kit8 (CCK-8) kit (Dojindo, Kumamoto, Japan) following the protocol. HCC827, PC-9, HCC827/gefitinib or PC-9/gefitinib cells $\left(5 \times 10^{3}\right.$ cell per well) were plated onto 96 -well plates overnight at $37^{\circ} \mathrm{C}$. After that, the culture medium was removed and the cells were treated with different concentrations of Tan IIA $(0,1,2,4,8$ or $16 \mu \mathrm{M})$ or gefitinib $(0,5,10$, $20,40,80,160$ or $320 \mathrm{nM}$ ) for $72 \mathrm{hrs}$ at $37^{\circ} \mathrm{C}$. Then, $10 \mu \mathrm{L}$ CCK-8 reagent was added into each well and incubated for another 2 hrs. Later on, a microplate reader (Bio-Tek Instruments Inc., Winooski, VT, USA) was used to measure the optical density (OD) of each well at $450 \mathrm{~nm}$.

\section{Combination Studies}

The combination index (CI) was used to calculate the drug combination studies by using Chou-Talalay method. ${ }^{23}$ HCC827/gefitinib or PC-9/gefitinib cells were exposed to solutions containing $0,10,20,40,80,160$ or $320 \mathrm{nM}$ gefitinib combined with Tan IIA $(2 \mu \mathrm{M})$. The CI value for the combination of gefitinib and TanIIA in NSCLC can be described as $\mathrm{CI}=\mathrm{DA} / \mathrm{ICx}, \mathrm{A}+\mathrm{DB} / \mathrm{ICx}, \mathrm{B} .^{24}$

\section{Colony Formation Assay}

HCC $827 /$ gefitinib cells $\left(5 \times 10^{3}\right.$ cells/well $)$ were plated into 6 -well plates overnight at $37^{\circ} \mathrm{C}$. After that, cells were treated with gefitinib $(40 \mathrm{nM})$ or/and Tan IIA $(2 \mu \mathrm{M})$ for another 3 days at $37^{\circ} \mathrm{C}$. Later on, cells were stained with methylene blue solution at room temperature for $1 \mathrm{hr}$. Then, 6-well plates were photographed using an Olympus IX71 fluorescence microscope (Olympus, Tokyo, Japan) and the number of cell colonies was counted.

\section{Immunofluorescence}

HCC 827/gefitinib or PC-9/gefitinib cells $\left(5 \times 10^{3}\right.$ cells/well) were plated into 6-well plates overnight at $37^{\circ} \mathrm{C}$. After that, the cells were treated with gefitinib or/and Tan IIA for $48 \mathrm{hrs}$ at $37^{\circ} \mathrm{C}$. Later on, the cells were fixed with $4 \%$ formaldehyde for 10 mins at room temperature, and then blocked with 5\% BSA for 30 mins at room temperature. After that, the cells were incubated with primary antibody against Ki67 (Santa Cruz Biotechnology, CA, USA) overnight at $4^{\circ} \mathrm{C}$. After washing with PBS three times, the cells were incubated with goat anti-rabbit secondary antibody (Santa Cruz) at 
$37^{\circ} \mathrm{C}$ for $1 \mathrm{hr}$. Later on, cell nuclei were stained with DAPI (Abcam Cambridge, MA, USA) for 5 mins. Images were captured using an Olympus IX71 fluorescence microscope.

\section{Flow Cytometry Assay}

Cell apoptosis analysis in gefitinib-resistant NSCLC cells was carried out using an Annexin V-FITC apoptosis detection kit (Thermo Fisher Scientific). HCC827/gefitinib cells were washed three times with pre-cold PBS, the cells were then stained with Annexin V-FITC and propidium iodide (PI, Thermo Fisher Scientific) for 20 mins in the dark. The apoptotic cells were counted using a FACS Calibur flow cytometer (BD Biosciences, Franklin Lakes, NJ, USA).

\section{Western Blot Assay}

Cells were lysed using RIPA Lysis Buffer (Thermo Fisher Scientific) at $4^{\circ} \mathrm{C}$. Proteins (30 $\mu$ g per lane) were separated on $10 \%$ SDS-PAGE gel, and then transferred onto a polyvinylidene fluoride membrane (PVDF; Millipore, Billerica, MA, USA). Following this, the membrane was blocked with 5\% non-fat milk for $1 \mathrm{hr}$ at room temperature. Later on, the membrane was incubated with primary antibodies overnight at $4^{\circ} \mathrm{C}$. Anti-cleaved caspase 3 (1:1000, Abcam Cambridge, MA, USA), anti-p-EGFR (1:1000, Abcam), anti-EGFR (1:1000, Abcam), anti-p-VEGFR2 (1:1000, Abcam), antiVEGFR2 (1:1000, Abcam), anti-p-Akt (1:1000, Abcam), anti-Akt (1:1000, Abcam), anti- $\beta$-actin (1:1000, Abcam). After that, the membranes were incubated with HRP-goat anti-rabbit secondary antibody (1: 5000, Abcam) for $1 \mathrm{hr}$ at room temperature. Enhanced chemiluminescence detection reagent (Tanon, Shanghai, China) was used to visualize the signal strength of the bands.

\section{Transwell Assay}

Cell migration and cell invasion assays were conducted using transwell chambers (Corning New York, NY, USA). HCC 827/gefitinib or PC-9/gefitinib cells $\left(3 \times 10^{4}\right.$ cells in $200 \mu \mathrm{L}$ serum-free medium) were seed onto the upper Transwell chambers. Then, $600 \mu \mathrm{L}$ of DMEM containing $20 \%$ FBS was then added into the lower chambers. Twentyfour hours later, the non-migratory or non-invaded cells were swabbed by a cotton bud. The migratory or invaded cells on the lower membrane surface were fixed with $4 \%$ paraformaldehyde for 15 mins. After that, the cells on the lower membrane surface were stained with $0.1 \%$ crystal violet for 20 mins. Five random fields were captured and counted under a fluorescence microscope. For transwell invasion assay, transwell chambers were pre-covered with Matrigel (BD Bioscience) before the experiments.

\section{Animal Study}

Male BALB/c nude mice ( $\mathrm{n}=16,4-6$ weeks, $18-20 \mathrm{~g}$ ) were obtained from Shanghai SLAC Laboratory Animal Co. Ltd., (Shanghai, China) in accordance with the Institutional Animal Care and Use Committee (IACUC) guidelines. HCC $827 /$ gefitinib cells $\left(5 \times 10^{6}\right.$ cells $)$ were suspended in $50 \mu \mathrm{L}$ of PBS, and injected subcutaneously into the right flanks of nude mice. When the tumor volume reached about $200 \mathrm{~mm}^{3}$, the mice were randomized into four groups: Vehicle group, $150 \mathrm{mg} / \mathrm{kg}$ gefitinib group, $20 \mathrm{mg} / \mathrm{kg}$ Tan IIA group, and gefitinib + Tan IIA group. Tumor formation was measured with vernier caliper once a week. Tumor volume was calculated by the formula: Volume $\left(\mathrm{mm}^{3}\right)=$ $\left(\right.$ Length $\times$ Width $\left.^{2}\right) / 2$. After 3 weeks, animals were sacrificed under anesthesia by following the recommended procedures of National Institutes of Health guide for the care and use of laboratory animals. All the experiments were approved by the Animal Experimental Ethics Committee of the Third Affiliated Hospital of Chongqing Medical University.

\section{Statistical Analysis}

GraphPad Prism 7 (GraphPad Software, Inc., La Jolla, CA, USA) was performed for statistical analysis. Data were represented as mean \pm standard deviation (SD). All experiments were repeated at least in three times. The comparisons among multiple groups were made with one-way analysis of variance (ANOVA) followed by Tukey's test. $\mathrm{P}<0.05$ was accepted as a statistically significant difference.

\section{Results}

\section{Tan IIA Enhances The Cytotoxic Effect Of Gefitinib In Gefitinib-Resistance NSCLC Cells}

The chemical structure of Tan IIA was indicated in Figure 1A. To compare the viability rate between the gefitinib-sensitive and gefitinib-resistance NSCLC cells, HCC827, HCC827/ gefitinib cells, and PC-9, PC-9/gefitinib cells were cultured in medium containing with different concentrations of gefitinib for 72 hrs. As shown in Figure 1B and C, the viability of HCC 827 and PC-9 cells was markedly lower than those of HCC827/gefitinib and PC-9/gefitinib cells, respectively. In addition, $2 \mu \mathrm{M}$ Tan IIA slightly inhibited the proliferation of HCC $827 / g e f i t i n i b$ and PC-9/gefitinib cells, and exhibited about $18 \%$ and $17 \%$ growth inhibition, respectively 
A<smiles>Cc1coc2c1C(=O)C(=O)c1c-2ccc2c1CCCC2(C)C</smiles>

Chemical structure of tanshinone IIA

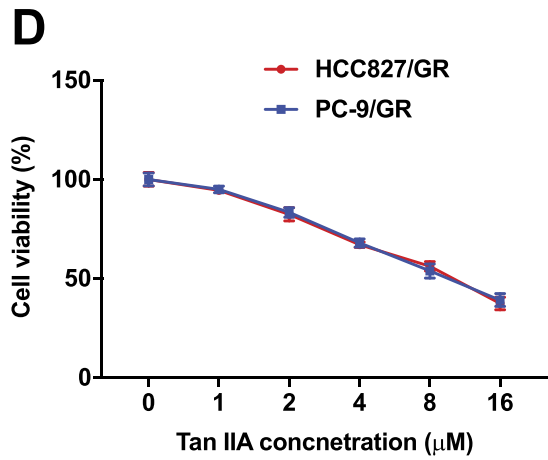

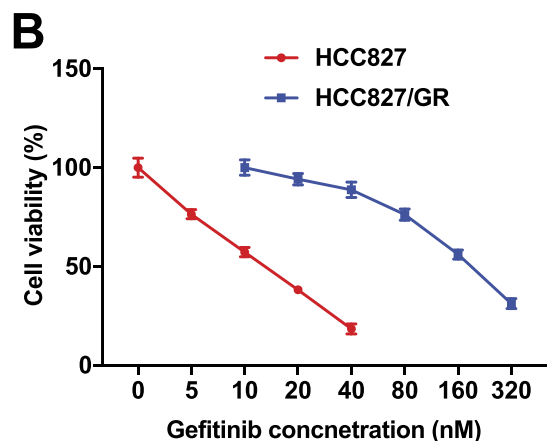

E

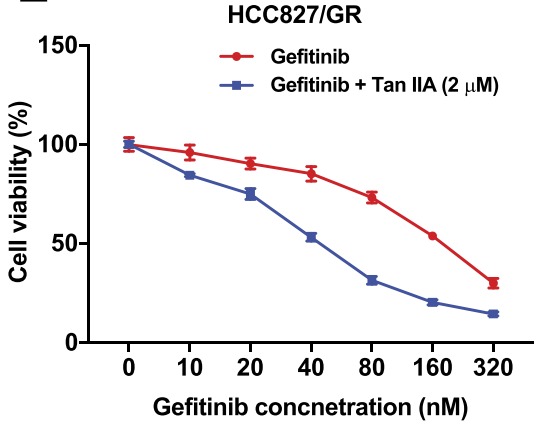

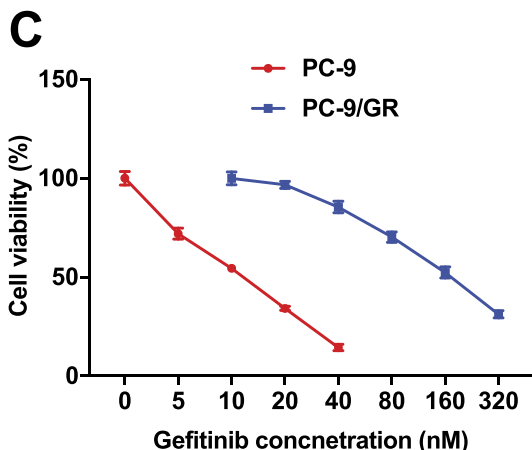

$\mathbf{F}$

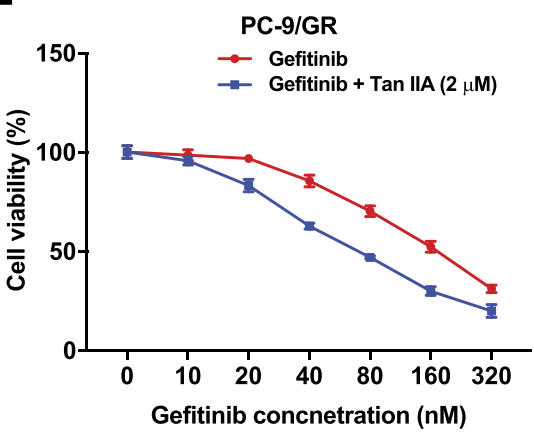

Figure I Tan IIA enhances the cytotoxic effect of gefitinib in gefitinib-resistance NSCLC cells. (A) The chemical structure of Tan IIA. (B) CCK-8 assay was used to detect the viability of NSCLC cells. HCC827 or HCC827/gefitinib cells were treated with $0,5,10,20,40,80,160$ or 320 nM gefitinib for 72 hrs, respectively. (C) PC-9, PC-9/ gefitinib cells were treated with $0,5,10,20,40,80,160$ or $320 \mathrm{nM}$ gefitinib for 72 hrs, respectively. (D) HCC827/gefitinib or PC-9/gefitinib cells were treated with 0, I, 2, 4, 6, 8 or $16 \mu \mathrm{M}$ Tan IIA for 72 hrs, respectively. (E) HCC827/gefitinib cells were treated with $40 \mathrm{nM}$ OXA, or $40 \mathrm{nM}$ OXA plus $2 \mu \mathrm{M}$ Tan IIA for 72 hrs. (F) PC-9/gefitinib cells were treated with $40 \mathrm{nM}$ OXA, or $40 \mathrm{nM}$ OXA plus $2 \mu \mathrm{M}$ Tan IIA for 72 hrs.

(Figure 1D). CCK-8 assay was further used to investigate the role of combination of gefitinib and Tan IIA on proliferation of gefitinib-resistance NSCLC cells. The cell viability in HCC827 cells showed no significant differences between the gefitinib treatment group and combination treatment group (Supplementary Figure 1A). However, as indicated in Figure $1 \mathrm{E}$ and $\mathrm{F}$, combined gefitinib with Tan IIA significantly inhibited the viability of HCC827/gefitinib and PC-9/gefitinib cells, compared with gefitinib treatment group.

Furthermore, as shown in Table $1, \mathrm{IC}_{50}$ value of gefitinib (alone treatment) was $172.20 \mathrm{nM}$ and $167.00 \mathrm{nM}$ in HCC827/ gefitinib and PC-9/gefitinib cells, respectively. However, when gefitinib was combined with Tan IIA $(2 \mu \mathrm{M})$, the $\mathrm{IC}_{50}$ value of gefitinib was decreased to $43.37 \mathrm{nM}$ and $75.77 \mathrm{nM}$, respectively. In addition, the CI value of gefitinib combined with Tan
IIA in HCC827/gefitinib cells was less than 0.6, which indicated the synergism effect (Table 1). Moreover, the CI value of gefitinib combined with Tan IIA in PC9/gefitinib cells was less than 0.8 , which indicated the moderate synergism effect (Table 1). These results suggested that combination of gefitinib with Tan IIA synergistically inhibited the proliferation of gefitinib-resistant NSCLC cells.

In particular, $2 \mu \mathrm{M}$ Tan IIA combined with $40 \mathrm{nM}$ gefitinib significantly inhibited the proliferation of HCC827/gefitinib and PC-9/gefitinib cells, and exhibited about $47 \%$ and $37 \%$ growth inhibition in HCC $827 /$ gefitinib and PC-9/gefitinib cells, respectively. Therefore, $40 \mathrm{nM}$ gefitinib were utilized in the following experiments. These data indicated that Tan IIA could enhance the cytotoxic effect of gefitinib in gefitinib-resistant NSCLC cells.

Table I Evaluation Of Combination Of Gefitinib With Tan IIA In Gefitinib-Resistant NSCLC Cells (72 HRS treatment)

\begin{tabular}{|l|l|l|l|l|}
\hline \multirow{2}{*}{ Drug Combination } & \multicolumn{2}{l|}{ HCC827/Gefitinib } & \multicolumn{2}{l|}{ PC-9/Gefitinib } \\
\cline { 2 - 5 } & IC 50 Value Of Gefitinib & CI Values & IC $\mathbf{5 0}$ Value Of Gefitinib & CI Values \\
\hline $\begin{array}{l}\text { Gefitinib (range 0 from } 320 \mathrm{nM} \text { ) } \\
\text { Gefitinib }+2 \mu \mathrm{M} \text { Tan IIA }\end{array}$ & IC50 $=172.20 \pm 1.21 \mathrm{nM}$ & - & IC50 $=167.00 \pm 1.12 \mathrm{nM}$ & - \\
IC50 $=42.37 \pm 1.82 \mathrm{nM}$ & 0.45 & IC50 $75.77 \pm 1.12 \mathrm{nM}$ & 0.66 \\
\hline
\end{tabular}




\section{Tan IIA Enhances The Anti-Proliferation Effect Of Gefitinib In Gefitinib-Resistant NSCLC Cells}

To investigate the role of Tan IIA and gefitinib on the proliferation of gefitinib-resistant NSCLC cells, colony formation and $\mathrm{Ki} 67$ immunofluorescence staining assays were used. As indicated in Figure 2A-D, gefitinib significantly inhibited the proliferation of HCC827/gefitinib cells, compared with the control group. As expected, Tan IIA markedly enhanced the anti-proliferation effect of gefitinib on HCC827/gefitinib cells. In addition, the anti-proliferation effect of Tan IIA combined with gefitinib was confirmed by testing PC9/gefitinib cells (Supplementary Figure 2A and B). These data indicated that Tan IIA could enhance the anti-proliferation effect of gefitinib in gefitinib-resistant NSCLC cells.

\section{Tan IIA Enhances The Pro-Apoptotic Effect Of Gefitinib In Gefitinib-Resistant NSCLC Cells}

Next, the effect of Tan IIA combined with gefitinib on cell apoptosis was detected with flow cytometric assay. As shown in Figure 3A and B, Supplementary Figure 3A and $\underline{B}$, treatment of HCC827/gefitinib and PC9/gefitinib cells with gefitinib or Tan IIA alone significantly induced cell apoptosis compared with the control group. As expected, combination of gefitinib with Tan IIA induced a substantial fraction of apoptosis in gefitinib-resistant NSCLC cells, compared with gefitinib treatment group. In addition, gefitinib markedly increased the levels of cleaved caspase 3 in HCC827/gefitinib and PC9/gefitinib cells, which were further enhanced in the presence of Tan IIA (Figure 3C and D, Supplementary
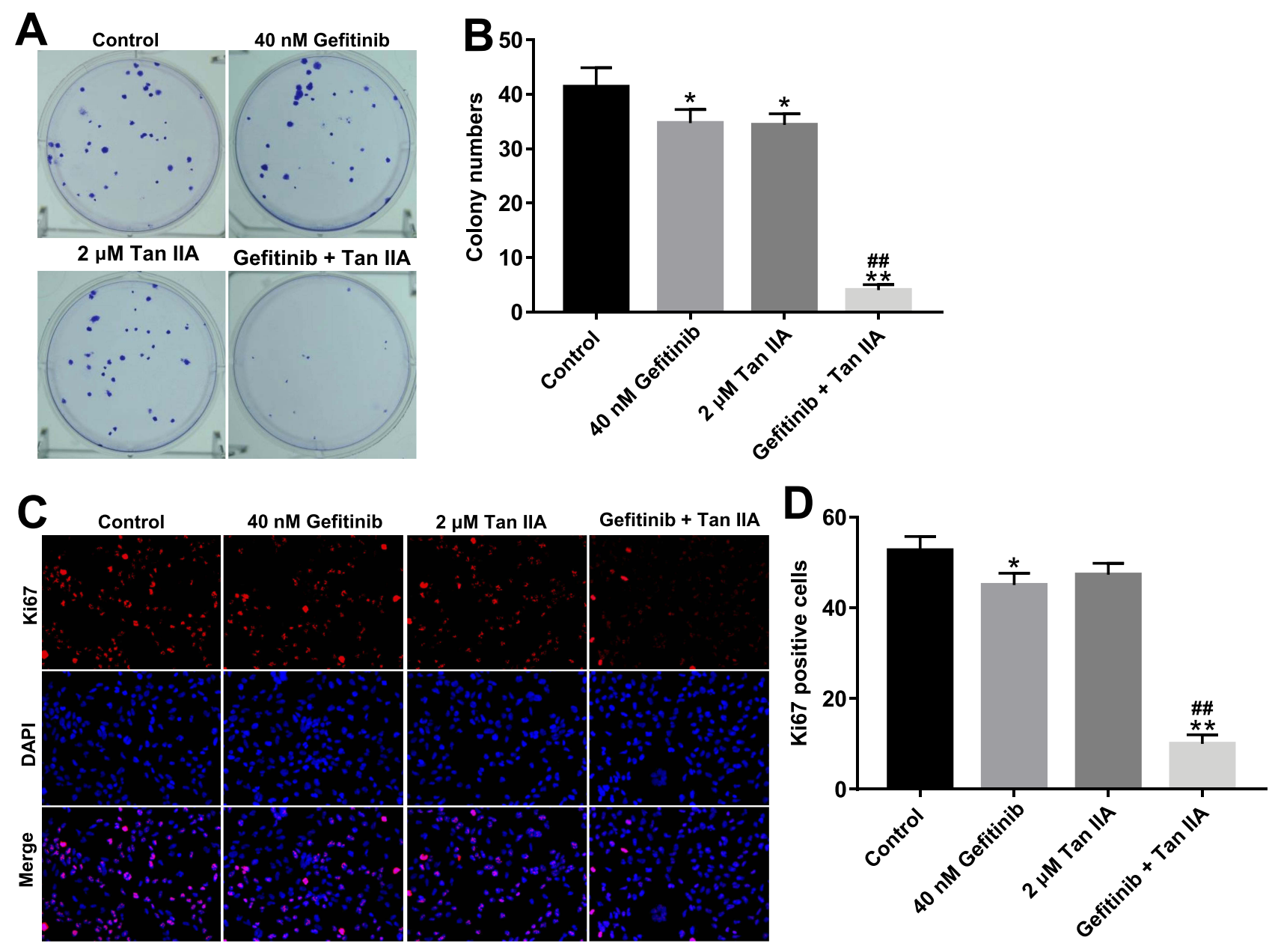

Figure 2 Tan IIA enhances the anti-proliferation effect of gefitinib in HCC827/gefitinib cells. (A, B) HCC827/gefitinib cells were treated with 40 nM OXA or/and $2 \mu M$ Tan IIA for $72 \mathrm{hrs}$. Colony formation assay was used to detect the colony formation of HCC827/gefitinib cells. (C, D) HCC827/gefitinib cells were treated with $40 \mathrm{nM}$ OXA or/ and $2 \mu \mathrm{M}$ Tan IIA for 48 hrs. Relative fluorescence expression levels were quantified by Ki67 and DAPI staining. *P<0.05, **P<0.0I compared with control group; ${ }^{\# \# P<0.0 I}$ compared with $40 \mathrm{nM}$ gefitinib group. 

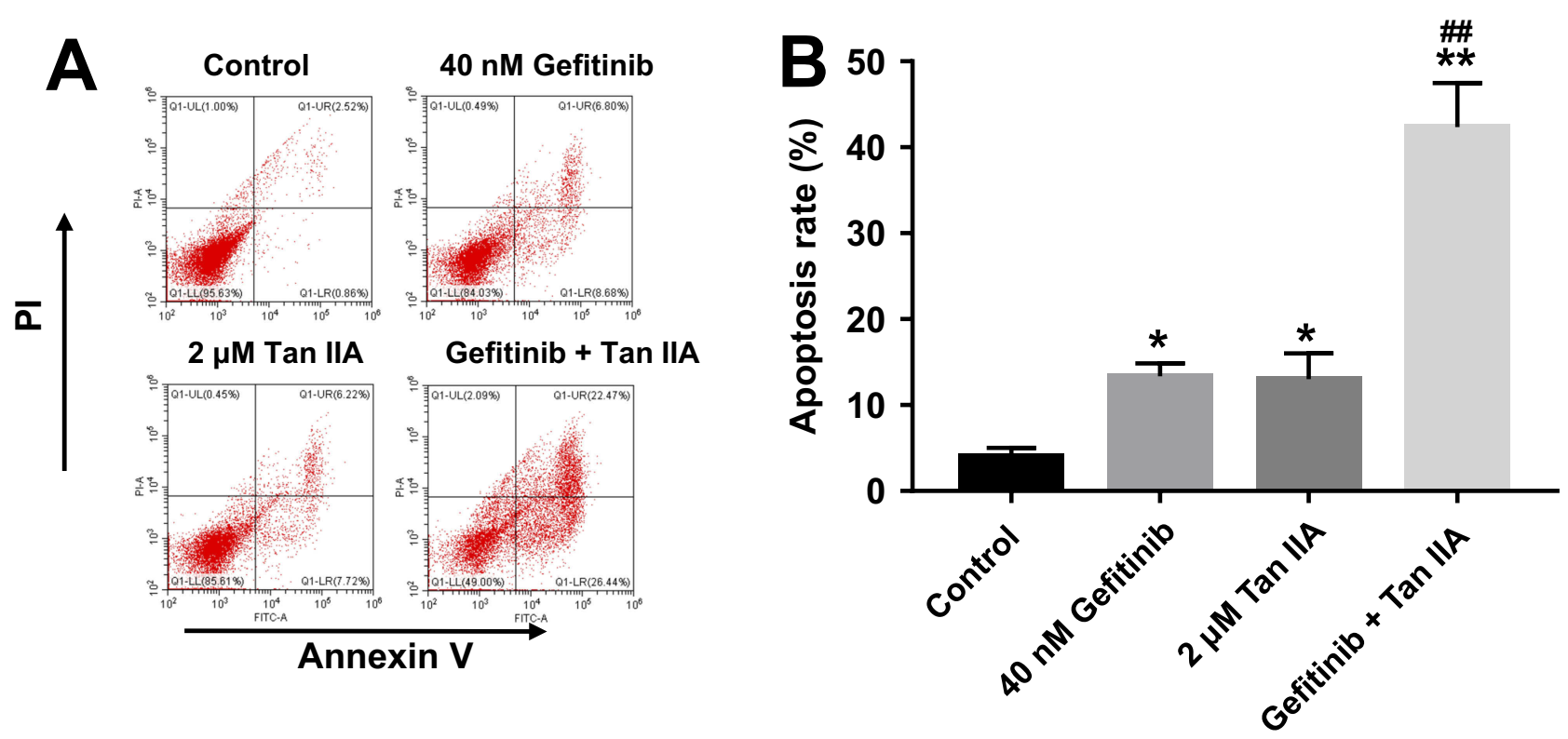

C
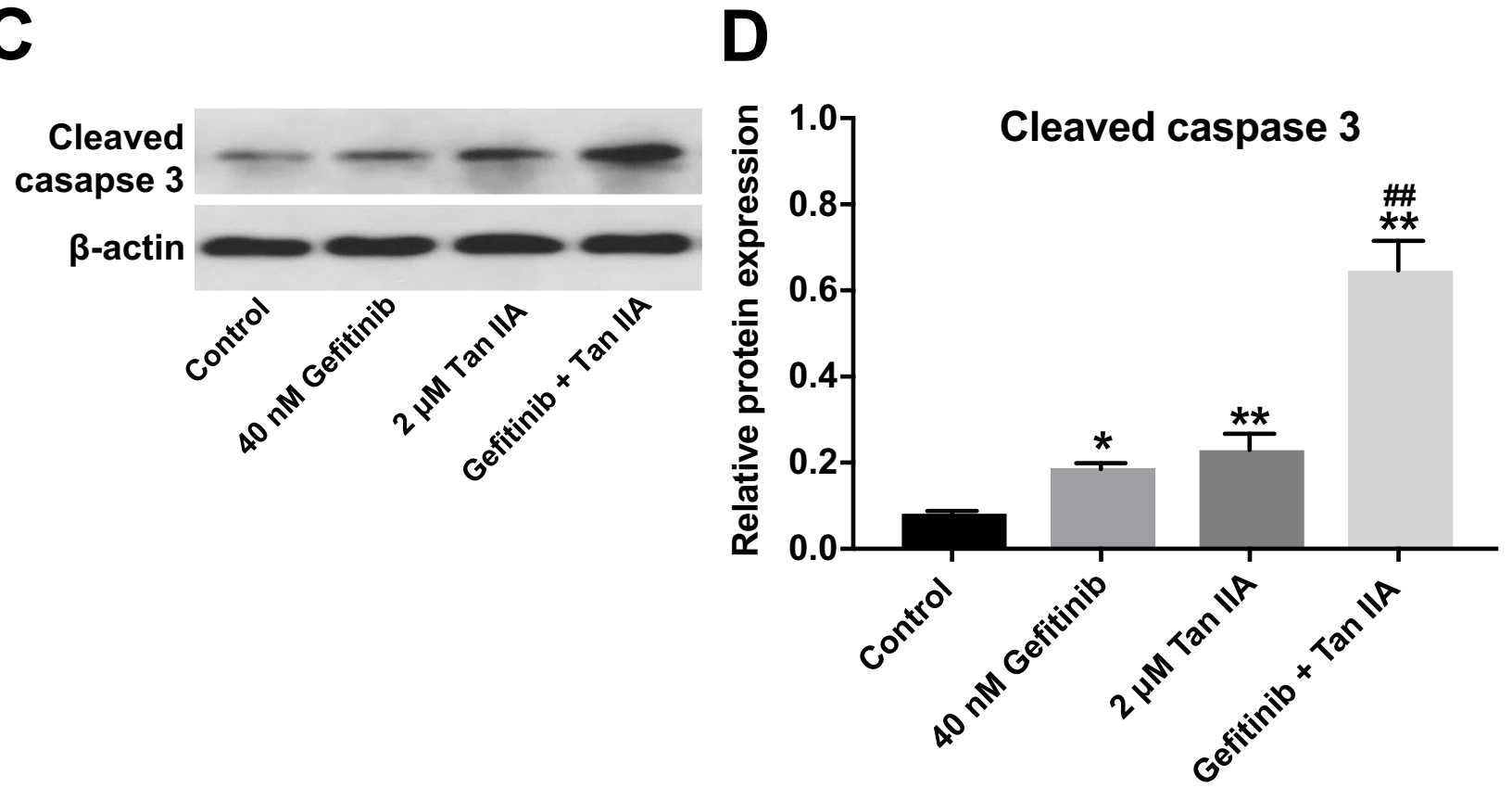

Figure 3 Tan IIA enhances the pro-apoptotic effect of gefitinib in HCC827/gefitinib cells. (A, B) HCC827/gefitinib cells were treated with 40 nM OXA or/and $2 \mu$ M Tan IIA for 72 hrs. Apoptotic cells were measured by flow cytometry. (C, D) Expression level of active caspase 3 in HCC827/gefitinib cells was detected with Western blotting. $* \mathrm{P}<0.05, * * \mathrm{P}<0.01$ compared with control group; ${ }^{\#} \mathrm{P}<0.01$ compared with $40 \mathrm{nM}$ gefitinib group.

Figure 3C and D). These results indicated that Tan IIA could enhance the pro-apoptotic effect of gefitinib in gefitinib-resistant NSCLC cells.

\section{Tan IIA Enhances The Inhibitory Effects Of Gefitinib On The Migration And Invasion Of Gefitinib-Resistant NSCLC Cells}

To further assess the role of Tan IIA and gefitinib on the migration and invasion of HCC827/gefitinib cells, transwell migration and invasion assays were applied. As indicated in
Figure 4A and B, Supplementary Figure 4A and B, gefitinib significantly inhibited the migration abilities of HCC827/gefitinib and PC9/gefitinib cells, which were further decreased in the presence of Tan IIA. In addition, combination of gefitinib with Tan IIA markedly inhibited the invasion ability of gefitinib-resistant NSCLC cells, compared with gefitinib treatment group (Figure 4C and D, Supplementary Figure 4C and D). These data suggested that Tan IIA could enhance the inhibitory effects of gefitinib on the migration and invasion of gefitinib-resistant NSCLC cells. 

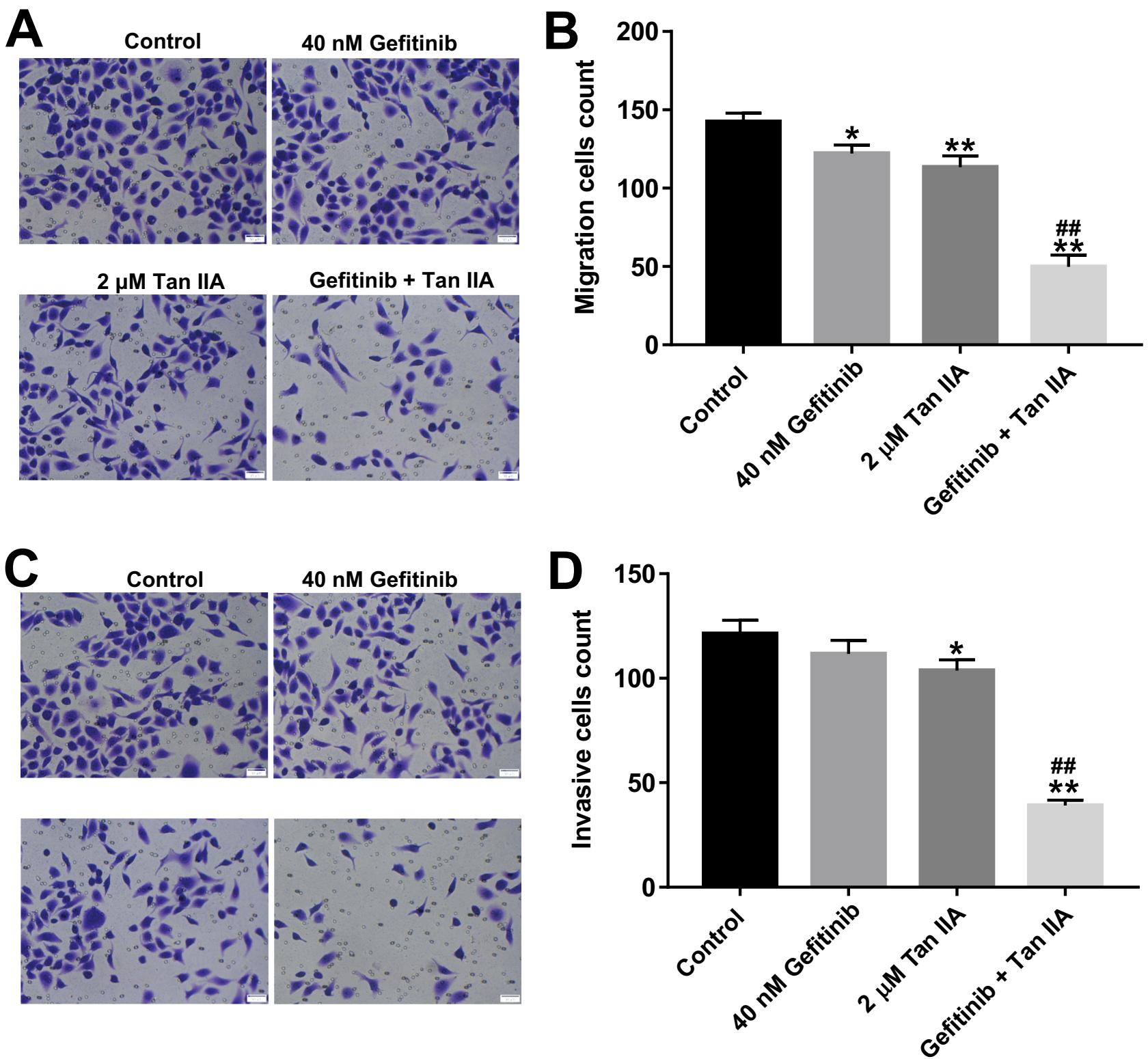

Figure 4 Tan IIA enhances the inhibitory effects of gefitinib on the migration and invasion of in HCC827/gefitinib cells. HCC827/gefitinib cells were treated with $40 \mathrm{nM}$ OXA or/and $2 \mu \mathrm{M}$ Tan IIA for 24 hrs. (A, B) Transwell migration assay was used to detect cell migration ability. (C, D) Transwell invasion assay was used to detect cell invasion ability. $* \mathrm{P}<0.05, * * \mathrm{P}<0.0$ I compared with control group; ${ }^{\#} \mathrm{P}<0.01$ compared with $40 \mathrm{nM}$ gefitinib group.

\section{Tan IIA Enhances The Sensitivity Of HCC827/Gefitinib Cells To Gefitinib By Regulation Of VEGFR2/Akt Pathway}

To assess the mechanism by which Tan IIA regulates gefitinib-resistance in NSCLC, the expressions of p-EGFR, p-VEGFR2 and p-Akt were measured by Western blotting assay. As indicated in Figure 5A and B, gefitinib markedly decreased the level of p-EGFR, while Tan IIA had no effects on the expression of p-EGFR in HCC827/gefitinib cells. As expected, combination of gefitinib and Tan IIA obviously downregulated the level of p-EGFR in HCC827/ gefitinib cells, which was reversed in the presence of VEGF (Figure 5A and B). In addition, gefitinib or Tan IIA significantly decreased the expressions of p-VEGFR2 and p-Akt in HCC827/gefitinib cells, which were further enhanced by combination treatment. However, the situations were markedly reversed in the presence of VEGF (Figure 5A, C and D). These results illustrated that Tan IIA could enhance the sensitivity of HCC827/gefitinib cells to gefitinib by downregulation of the VEGFR/Akt pathway. 


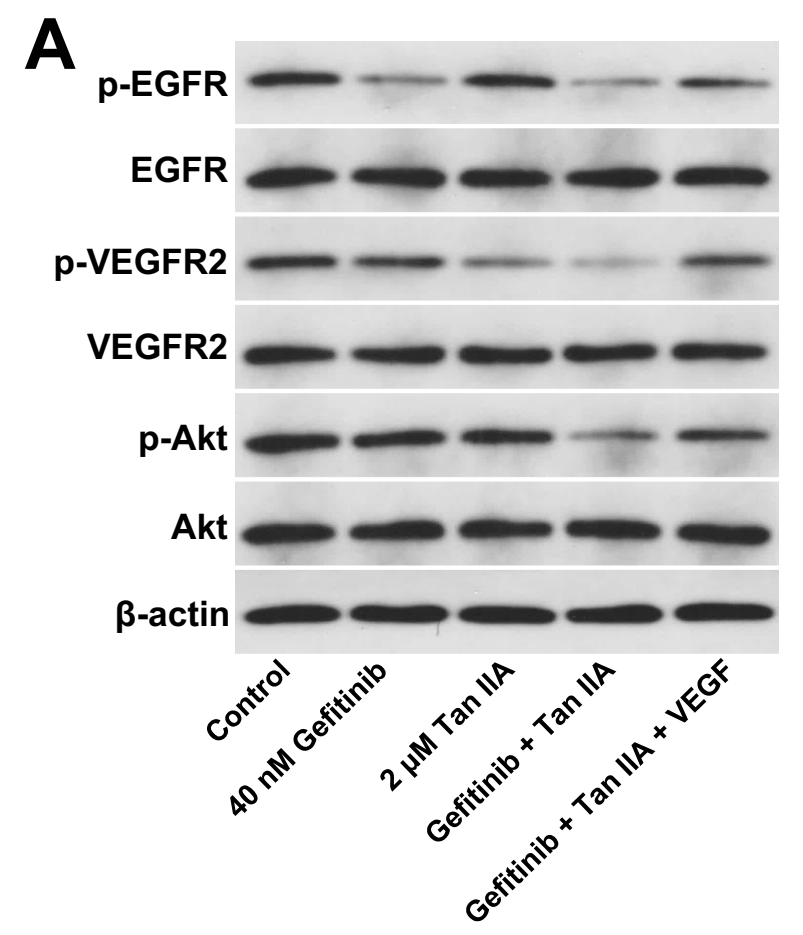

B
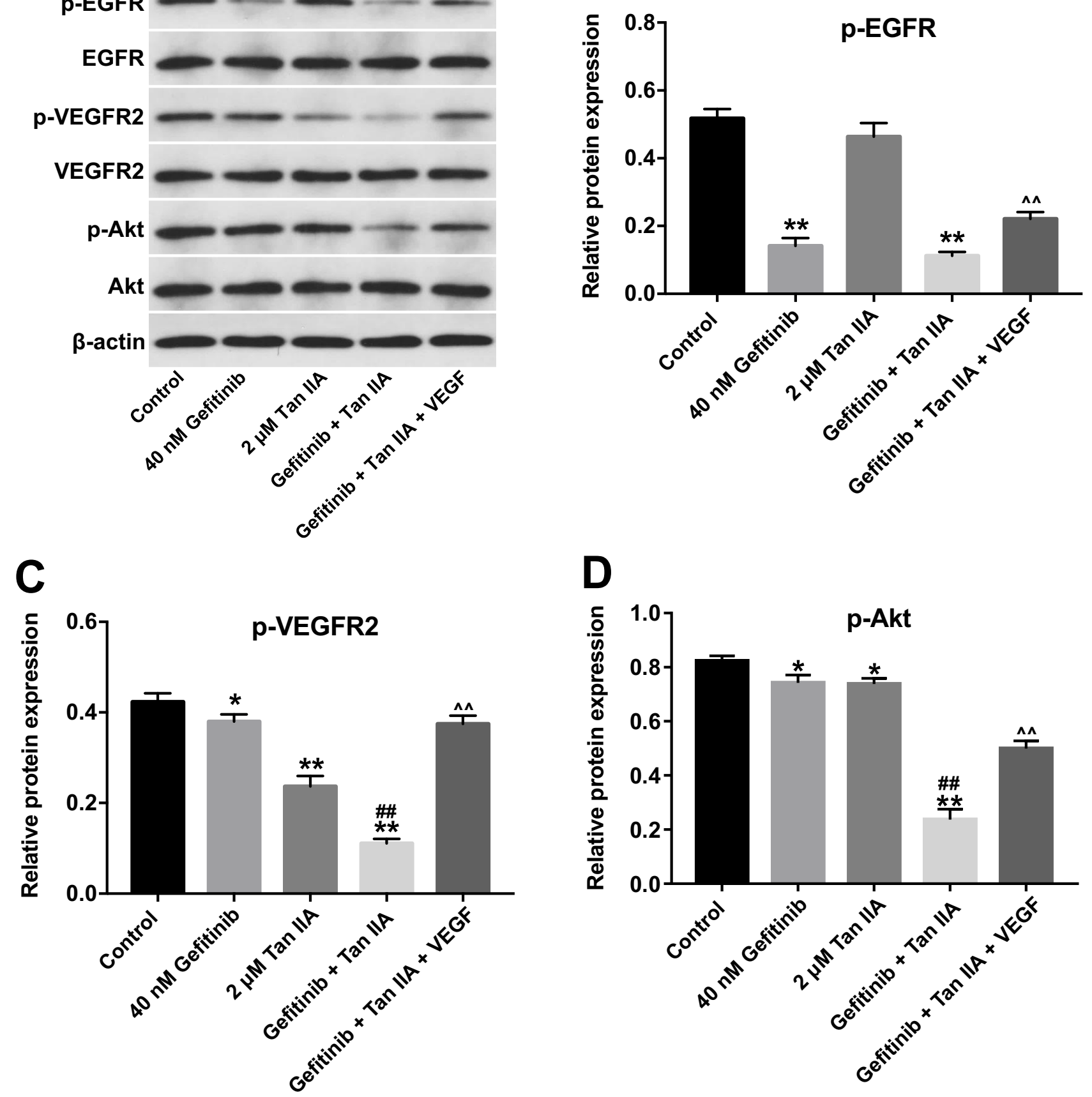

D

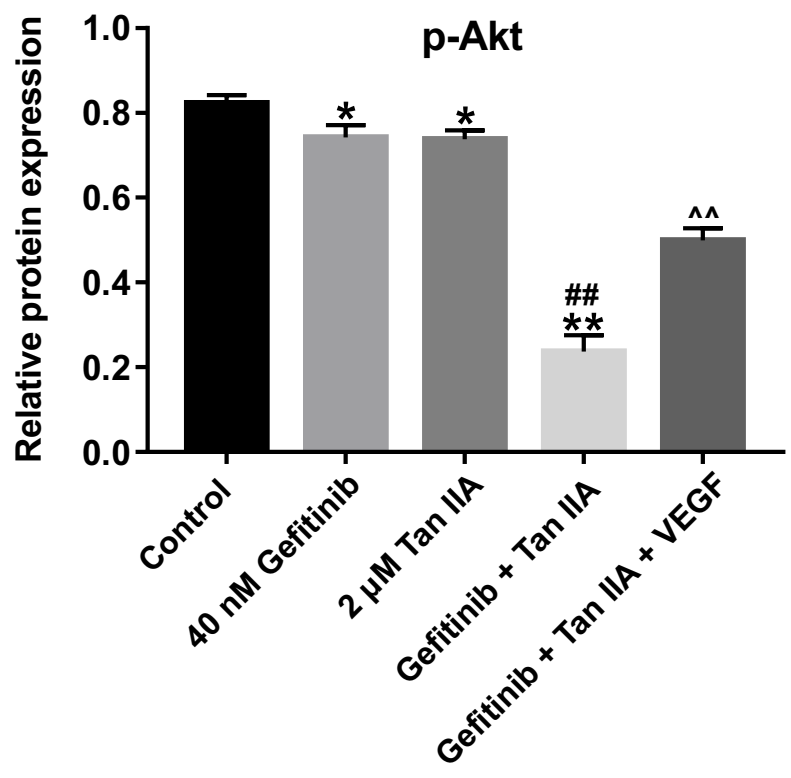

Figure 5 Tan IIA enhances the sensitivity of HCC827/gefitinib cells to gefitinib by regulation of VEGFR/Akt pathway. HCC827/gefitinib cells were treated with 40 nM OXA or/and $2 \mu \mathrm{M}$ Tan IIA, or OXA + Tan IIA + VEGF for 72 hrs. (A) Expression levels of p-EGFR, p-VEGFR2 and p-Akt in HCC827/gefitinib cells were detected with Western blotting. $\boldsymbol{\beta}$-actin was used as an internal control. (B-D) The relative expressions of $\mathrm{p}$-EGFR, $\mathrm{p}$-VEGFR2 and $\mathrm{p}$-Akt in HCC827/gefitinib cells were quantified via normalization to $\boldsymbol{\beta}$-actin. ${ }^{* P}<0.05$, ${ }^{* * P}<0.01$ compared with control group; ${ }^{\# \#} \mathrm{P}<0.01$ compared with $40 \mathrm{nM}$ gefitinib group. ${ }^{\wedge} \mathrm{P}<0.0 \mathrm{I}$ compared with gefitinib + Tan IIA group.

\section{Tan IIA Enhances The Sensitivity Of}

HCC827/Gefitinib Cells To Gefitinib In Vivo

Previous data indicated that Tan IIA could reverse gefitinibresistance in gefitinib-resistant NSCLC cells in vitro, then we further investigate the effect of Tan IIA combined with gefitinib in mouse xenograft model of HCC827/gefitinib.
As shown in Figure $6 \mathrm{~A}-\mathrm{C}$, gefitinib treatment had very limited effect on tumor volume and tumor weight. Meanwhile, Tan IIA treatment slightly inhibited the tumor volume and tumor weight. As expected, combination of gefitinib with Tan IIA significantly decreased the tumor volume and tumor weight, compared with gefitinib treatment 

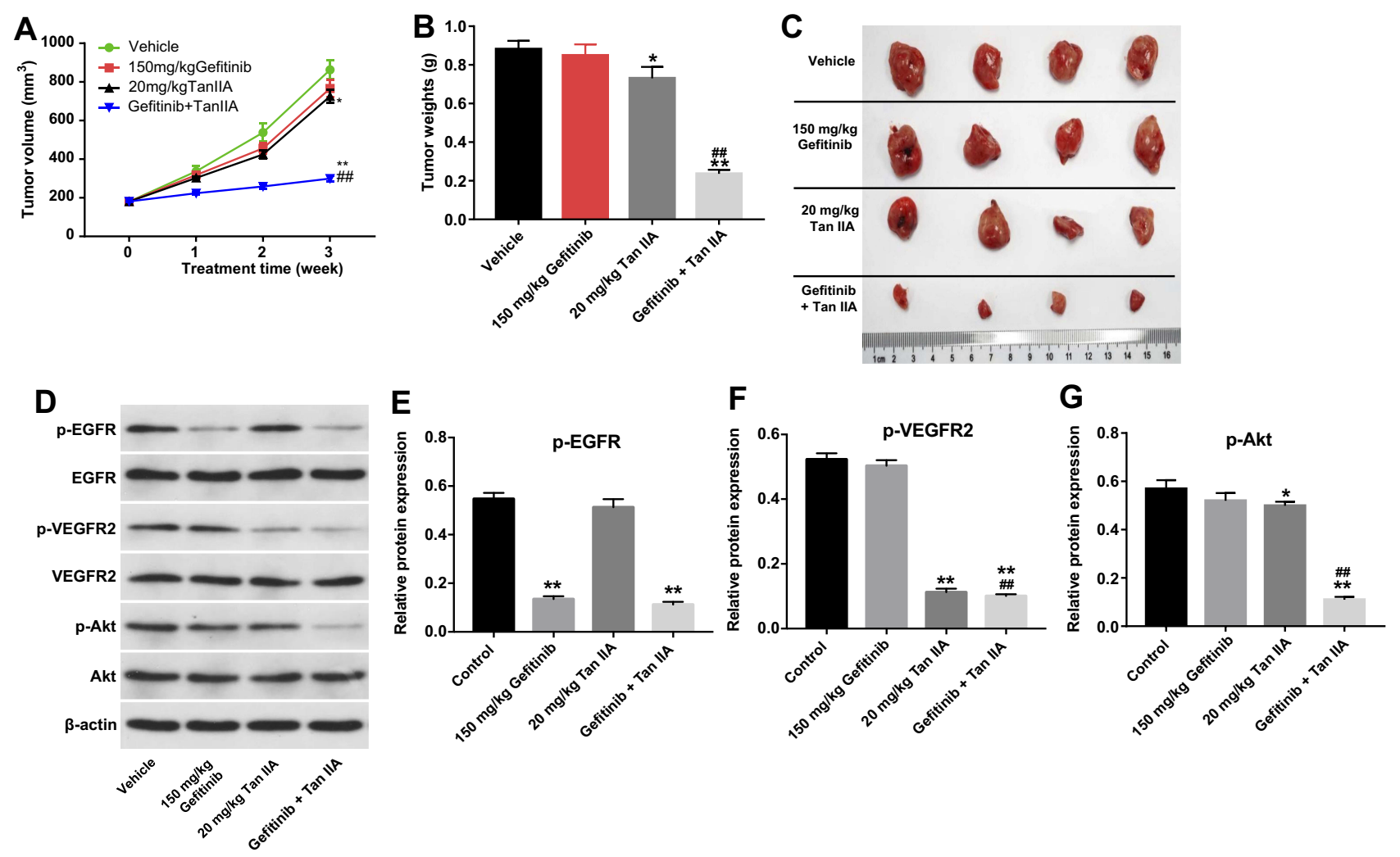

Figure 6 Tan IIA enhances the sensitivity of HCC827/gefitinib cells to gefitinib in vivo. HCC827/gefitinib cells were injected subcutaneously into nude mice to form subcutaneous tumors. (A) Tumor volumes of mice were measured weekly. (B) HCC827/gefitinib xenograft tumors were excised from xenografts and pictured on day $2 \mathrm{I}$. (C) The weight in each group of mice was calculated on day 21 . (D) Expression levels of p-EGFR, p-VEGFR2 and p-Akt in tumor tissues were measured with Western blotting. (E, F, G) The relative expressions of $\mathrm{p}$-EGFR, $\mathrm{p}$-VEGFR2 and $\mathrm{p}$-Akt were quantified via normalization to $\boldsymbol{\beta}$-actin. *P<0.05, **P<0.0I compared with control group; ${ }^{\prime \prime} \mathrm{P}<0.01$ compared with $150 \mathrm{mg} / \mathrm{kg}$ gefitinib group.

group (Figure 6A-C). In addition, gefitinib significantly decreased the expression of p-EGFR in tumor tissues (Figure 6D and E). Meanwhile, gefitinib had no effects on the expressions of p-VEGFR and p-Akt in tumor tissues, whereas the levels of p-VEGFR and p-Akt were markedly decreased in the presence of Tan IIA (Figure 6D, F and G). These results illustrated that combination of gefitinib with Tan IIA could inhibit tumorigenesis in HCC827/gefitinib xenograft by downregulation of the VEGFR/Akt pathway.

\section{Discussion}

Gefitinib, an EGFR-targeted tyrosine kinase inhibitor, has been used as a first-line drug for NSCLC. ${ }^{25}$ However, drug resistance is a primary obstacle in the response of NSCLC patients to gefitinib. ${ }^{26}$ Previous study indicated that finding new combinations of drugs might be a potential method to eliminate chemoresistance. ${ }^{27,28}$ In order to find a new therapy to enhance the sensitivity of NSCLC cells to gefitinib, gefitinib-resistant human NSCLC cells were used.
It has been shown that Tan IIA could enhance the sensitivity of cancer cells to chemotherapeutic drugs, such as doxorubicin, cisplatin. ${ }^{29,30} \mathrm{Li}$ et al found that Tan IIA could reverse doxorubicin resistance in doxorubicin-resistant breast cancer cells. ${ }^{31}$ Meanwhile, Tan IIA enhanced the sensitivity of lung cancers to cyclophosphamide via inducing apoptosis. ${ }^{32}$ In this study, we found that the inhibitory effects of gefitinib on the proliferation, migration and invasion of gefitinib-resistant NSCLC cells were enhanced in the presence of Tan IIA through inducing apoptosis. Chen et al indicated that combination of curcumin with gefitinib significantly induced apoptosis in gefitinib-resistant NSCLC cells, which was consistent with our studies. ${ }^{33}$ For the first time, we found that Tan IIA could reverse gefitinib-resistance in NSCLC. However, the mechanism underlying the chemo-preventive effect of Tan IIA has not been entirely illustrated.

It has been shown that gefitinib exhibited an inhibitory role on EGFR phosphorylation in NSCLC. ${ }^{34}$ In this study, gefitinib markedly decreased the level of p-EGFR, while Tan IIA had no effects on the phosphorylation level of 
EGFR in gefitinib-resistant NSCLC cells. In addition, combination treatment had no effects on the phosphorylation level of EGFR in gefitinib-resistant NSCLC cells as well, compared with gefitinib treatment group. Therefore, we speculated that EGFR signaling was not involved in combination-induced apoptosis of gefitinib-resistant NSCLC cells in vitro and in vivo.

Evidence indicated that VEGF, vascular endothelial growth factor, could stimulate cell proliferation and inhibit cell apoptosis in different cancer cells. ${ }^{35}$ It has been shown that VEGF exerts its biological activity in endothelial cells via combination with VEGFR2. ${ }^{35}$ Xie et al indicated that Tan IIA could induce apoptosis of NSCLC cells via decreasing VEGF/VEGFR2 expression. ${ }^{36} \mathrm{Su}$ et al found that Tan IIA inhibited the proliferation of gastric carcinoma cells by downregulation the expression of VEGFR. ${ }^{37}$ Meanwhile, apatinib could inhibit the proliferation of gefitinib-resistant NSCLC cells by inhibiting VEGFR signaling pathway. ${ }^{38}$ In the present study, our findings are in line with previous studies, showing that gefitinib or Tan IIA significantly downregulated the phosphorylation level of VEGFR2 in HCC827/ gefitinib cells, which were further enhanced by combination treatment. However, this situation was reversed by VEGF. These data suggested that Tan IIA enhanced the sensitivity of HCC827/gefitinib cells to gefitinib by downregulation of VEGFR2, which could exhibit an anti-angiogenic activity in gefitinib-resistant NSCLC cells.

Combination of VEGF and VEGFR2 could activate Akt pathway in endothelial cells, contributing to the promotion of cell proliferation. ${ }^{39}$ In this study, we found that combination treatment obviously downregulated the phosphorylation level of Akt in HCC827/gefitinib cells. Therefore, these data illustrated for the first time that combination of gefitinib with Tan IIA inhibited the proliferation of gefitinib-resistant NSCLC cells via inhibition of VEGFR signaling pathway, then indirectly inhibiting downstream Akt signaling pathway, thereby suppressing the tumorigenesis of NSCLC.

\section{Conclusion}

Collectively, Tan IIA reversed gefitinib-resistance in gefitinib-resistant NSCLC cells via inhibiting VEGFR/Akt pathway. Therefore, gefitinib combined with Tan IIA might be considered as a therapeutic approach for the treatment of gefitinib-resistant NSCLC.

\section{Disclosure}

The authors report no conflicts of interest in this work.

\section{References}

1. Sun T, Zhao Q, Zhang C, et al. Screening common signaling pathways associated with drug resistance in non-small cell lung cancer via gene expression profile analysis. Cancer Med. 2019. doi:10.1002/ cam4.2190

2. Travis WD, Brambilla E, Burke AP, Marx A, Nicholson AG. Introduction to the 2015 World Health Organization classification of tumors of the lung, pleura, thymus, and heart. J Thorac Oncol. 2015;10(9):1240-1242. doi:10.1097/JTO.0000000000000663

3. Ramalingam SS, Owonikoko TK, Khuri FR. Lung cancer: new biological insights and recent therapeutic advances. CA Cancer $J$ Clin. 2011;61(2):91-112. doi:10.3322/caac.v61:2

4. Planchard D, Popat S, Kerr K, et al. Metastatic non-small cell lung cancer: ESMO clinical practice guidelines for diagnosis, treatment and follow-up. Ann Oncol. 2018;29(Supplement_4):iv192-iv237.

5. Chang JY, Senan S, Paul MA, et al. Stereotactic ablative radiotherapy versus lobectomy for operable stage I non-small-cell lung cancer: a pooled analysis of two randomised trials. Lancet Oncol. 2015;16 (6):630-637. doi:10.1016/S1470-2045(15)70168-3

6. Hirsch FR, Scagliotti GV, Mulshine JL, et al. Lung cancer: current therapies and new targeted treatments. Lancet. 2017;389 (10066):299-311. doi:10.1016/S0140-6736(16)30958-8

7. Chang H, Qu J, Wang J, Liang X, Sun W. Circular RNA circ_0026134 regulates non-small cell lung cancer cell proliferation and invasion via sponging miR-1256 and miR-1287. Biomed Pharmacother. 2019;112:108743. doi:10.1016/j.biopha.2019.108743

8. Xian H, Feng W, Zhang J. Schizandrin A enhances the efficacy of gefitinib by suppressing IKKbeta/NF-kappaB signaling in non-small cell lung cancer. Eur J Pharmacol. 2019. doi:10.1016/j.ejphar.2019.04.016

9. Pennell NA, Lynch TJ Jr. Combined inhibition of the VEGFR and EGFR signaling pathways in the treatment of NSCLC. Oncologist. 2009;14(4):399-411. doi:10.1634/theoncologist.2008-0276

10. Liang W, Zheng Y, Zhang J, Sun X. Multiscale modeling reveals angiogenesis-induced drug resistance in brain tumors and predicts a synergistic drug combination targeting EGFR and VEGFR pathways. $B M C$ Bioinformatics. 2019;20(Suppl 7):203. doi:10.1186/s12859-019-2737-1

11. Zhang HQ, Gong FH, Ye JQ, et al. Design and discovery of 4anilinoquinazoline-urea derivatives as dual TK inhibitors of EGFR and VEGFR-2. Eur J Med Chem. 2017;125:245-254. doi:10.1016/j. ejmech.2016.09.039

12. Barbosa ML, Lima LM, Tesch R, et al. Novel 2-chloro-4-anilinoquinazoline derivatives as EGFR and VEGFR-2 dual inhibitors. Eur $J$ Med Chem. 2014;71:1-14. doi:10.1016/j.ejmech.2013.10.058

13. Jotte RM, Spigel DR. Advances in molecular-based personalized non-small-cell lung cancer therapy: targeting epidermal growth factor receptor and mechanisms of resistance. Cancer Med. 2015;4 (11):1621-1632. doi:10.1002/cam4.506

14. Gefitinib delays NSCLC recurrence after surgery. Cancer Discov. 2017;7(7):Of4. doi:10.1158/2159-8290.CD-NB2017-076

15. Thress KS, Paweletz CP, Felip E, et al. Acquired EGFR C797S mutation mediates resistance to AZD9291 in non-small cell lung cancer harboring EGFR T790M. Nat Med. 2015;21(6):560-562. doi: $10.1038 / \mathrm{nm} .3854$

16. Tang JC, Ren YG, Zhao J, et al. Shikonin enhances sensitization of gefitinib against wild-type EGFR non-small cell lung cancer via inhibition PKM2/stat3/cyclinD1 signal pathway. Life Sci. 2018;204:71-77. doi:10.1016/j.lfs.2018.05.012

17. Quan M, Lv Y, Dai Y, et al. Tanshinone IIA protects against lipopolysaccharide-induced lung injury through targeting Sirt1. J Pharm Pharmacol. 2019. doi:10.1111/jphp.13087

18. Meng Z, Si CY, Teng S, Yu XH, Li HY. Tanshinone IIA inhibits lipopolysaccharide induced inflammatory responses through the TLR4/TAK1/NFkappaB signaling pathway in vascular smooth muscle cells. Int J Mol Med. 2019;43(4):1847-1858. doi:10.3892/ ijmm.2019.4100 
19. Qi D, Wang M, Zhang D, Li H. Tanshinone IIA protects lens epithelial cells from $\mathrm{H} 2 \mathrm{O} 2$-induced injury by upregulation of lncRNA ANRIL. J Cell Physiol. 2019. doi:10.1002/jcp.v234.9

20. Zhang X, Zhou Y, Gu YE. Tanshinone IIA induces apoptosis of ovarian cancer cells in vitro and in vivo through attenuation of PI3K/AKT/JNK signaling pathways. Oncol Lett. 2019;17(2):18961902. doi:10.3892/ol.2018.9744

21. Liu F, Yu G, Wang G, et al. An NQO1-initiated and p53-independent apoptotic pathway determines the anti-tumor effect of tanshinone IIA against non-small cell lung cancer. PLoS ONE. 2012;7(7):e42138. doi:10.1371/journal.pone.0042138

22. Xie J, Liu JH, Liu H, et al. Tanshinone IIA combined with adriamycin inhibited malignant biological behaviors of NSCLC A549 cell line in a synergistic way. BMC Cancer. 2016;16(1):899. doi:10.1186/s12885-0162921-x

23. Chou TC, Talalay P. Quantitative analysis of dose-effect relationships: the combined effects of multiple drugs or enzyme inhibitors. Adv Enzyme Regul. 1984;22:27-55. doi:10.1016/0065-2571(84)90007-4

24. Chen S, Zhang Z, Zhang J. Emodin enhances antitumor effect of paclitaxel on human non-small-cell lung cancer cells in vitro and in vivo. Drug Des Devel Ther. 2019;13:1145-1153. doi:10.2147/DDDT.S196319

25. Li B, Yao Z, Wan Y, Lin D. Overexpression of OCT4 is associated with gefitinib resistance in non-small cell lung cancer. Oncotarget. 2016;7(47):77342-77347. doi:10.18632/oncotarget.12999

26. Liu W, Ning J, Li C, et al. Overexpression of Sphk2 is associated with gefitinib resistance in non-small cell lung cancer. Tumour Biol. 2016;37(5):6331-6336. doi:10.1007/s13277-015-4480-1

27. Sooman L, Gullbo J, Bergqvist M, et al. Synergistic effects of combining proteasome inhibitors with chemotherapeutic drugs in lung cancer cells. BMC Res Notes. 2017;10(1):544. doi:10.1186/ s13104-017-2842-z

28. Gualdani R, de Clippele M, Ratbi I, Gailly P, Tajeddine N. Storeoperated calcium entry contributes to cisplatin-induced cell death in non-small cell lung carcinoma. Cancers (Basel). 2019;11(3):430. doi: $10.3390 /$ cancers 11030430

29. Li K, Liu W, Zhao Q, et al. Combination of tanshinone IIA and doxorubicin possesses synergism and attenuation effects on doxorubicin in the treatment of breast cancer. Phytother Res. 2019.
30. Zhao YX, Luo D, Zhang YH, et al. [The effect of tanshinone A potentiates the effects of Cisplatin in Fadu cells in vitro through downregulation of survivin]. Lin Chung Er Bi Yan Hou Tou Jing Wai Ke Za Zhi. 2017;31(10):781-784. doi:10.13201/j.issn.10011781.2017.10.011

31. Li K, Lai H. TanshinoneIIA enhances the chemosensitivity of breast cancer cells to doxorubicin through down-regulating the expression of MDR-related ABC transporters. Biomed Pharmacother. 2017;96:371-377.

32. Li Q, Hu K, Tang S, Xu LF, Luo YC. Anti-tumor activity of tanshinone IIA in combined with cyclophosphamide against Lewis mice with lung cancer. Asian Pac J Trop Med. 2016;9(11):1084-1088. doi:10.1016/j.apjtm.2016.09.003

33. Chen P, Huang HP, Wang Y, et al. Curcumin overcome primary gefitinib resistance in non-small-cell lung cancer cells through inducing autophagy-related cell death. J Exp Clin Cancer Res. 2019;38 (1):254. doi:10.1186/s13046-019-1234-8

34. Nguyen KS, Neal JW. First-line treatment of EGFR-mutant nonsmall-cell lung cancer: the role of erlotinib and other tyrosine kinase inhibitors. Biologics. 2012;6:337-345. doi:10.2147/BTT.S26558

35. Shibuya M. VEGF-VEGFR signals in health and disease. Biomol Ther (Seoul). 2014;22(1):1-9. doi:10.4062/biomolther.2013.113

36. Xie J, Liu J, Liu H, et al. The antitumor effect of tanshinone IIA on anti-proliferation and decreasing VEGF/VEGFR2 expression on the human non-small cell lung cancer A549 cell line. Acta Pharm Sin B. 2015;5(6):554-563. doi:10.1016/j.apsb.2015.07.008

37. Su CC. Tanshinone IIA inhibits gastric carcinoma AGS cells by decreasing the protein expression of VEGFR and blocking Ras/Raf/ MEK/ERK pathway. Int $J$ Mol Med. 2018;41(4):2389-2396. doi:10.3892/ijmm.2018.3407

38. Song YA, Ma T, Zhang XY, et al. Apatinib preferentially inhibits PC9 gefitinib-resistant cancer cells by inducing cell cycle arrest and inhibiting VEGFR signaling pathway. Cancer Cell Int. 2019;19:117. doi:10.1186/s12935-019-0836-8

39. Abid MR, Guo S, Minami T, et al. Vascular endothelial growth factor activates PI3K/Akt/forkhead signaling in endothelial cells. Arterioscler Thromb Vasc Biol. 2004;24(2):294-300. doi:10.1161/ 01.ATV.0000110502.10593.06
OncoTargets and Therapy

\section{Publish your work in this journal}

OncoTargets and Therapy is an international, peer-reviewed, open access journal focusing on the pathological basis of all cancers, potential targets for therapy and treatment protocols employed to improve the management of cancer patients. The journal also focuses on the impact of management programs and new therapeutic
Dovepress

agents and protocols on patient perspectives such as quality of life, adherence and satisfaction. The manuscript management system is completely online and includes a very quick and fair peer-review system, which is all easy to use. Visit http://www.dovepress.com/ testimonials.php to read real quotes from published authors. 